\title{
Visibility Representation of Plane Graphs with Simultaneous Bound for Both Width and Height
}

\author{
Jiun-Jie Wang! Xin $\mathrm{He}^{1}$
}

1 Department of Computer Science and Engineering, State University of New York at Buffalo, Buffalo, NY 14260

\begin{abstract}
The visibility representation (VR for short) is a classical representation of plane graphs. It has various applications and has been extensively studied. A main focus of the study is to minimize the size of the VR. The trivial upper bound is $(n-1) \times(2 n-5)$ (height $\times$ width). It is known that there exists a plane graph $G$ with $n$ vertices where any VR of $G$ requires a grid of size at least $\frac{2}{3} n \times\left(\frac{4}{3} n-3\right)$. For upper bounds, it is known that every plane graph has a VR with grid size at most $\frac{2}{3} n \times(2 n-5)$, and a VR with grid size at most $(n-1) \times \frac{4}{3} n$. It has been an open problem to find a VR with both height and width simultaneously bounded away from the trivial upper bounds (namely with size at most $c_{h} n \times c_{w} n$ with $c_{h}<1$ and $\left.c_{w}<2\right)$.

In this paper, we provide the first VR construction with this property. We prove that every plane graph of $n$ vertices has a VR with height at most $\frac{23}{24} n+2\lceil\sqrt{n}\rceil+10$ and width at most $\frac{23}{12} n$. The area of our VR is larger than the area of some of the previous results. However, bounding one dimension of the VR only requires finding a good st-orientation or a good dual $s^{*} t^{*}$-orientation of $G$. On the other hand, to bound both dimensions of VR simultaneously, one must find a good st-orientation and a good dual $s^{*} t^{*}$-orientation at the same time, which is far more challenging. Our VR algorithm is based on an st-orientation of plane graphs with special properties. Since st-orientations are a very useful concept in other applications, this result may be of independent interests.
\end{abstract}

\begin{tabular}{|cccc|}
\hline Submitted: & Reviewed: & Revised: & Accepted: \\
May 2011 & November 2011 & February 2012 & April 2012 \\
& Final: & Published: & \\
& April 2012 & May 2012 \\
Article type: & Communicated by: \\
Regular paper & A. Symvonis \\
\hline
\end{tabular}

Research supported in part by NSF Grant CCR-0635104.

E-mail addresses: jiunjiew@buffalo.edu (Jiun-Jie Wang) xinhe@buffalo.edu (Xin He) 


\begin{tabular}{|c|c|c|c|c|}
\hline \hline & \multicolumn{2}{|c|}{ Plane Graph } & \multicolumn{2}{c|}{ 4-Connected Plane Graph } \\
\hline & Width & Height & Width & Height \\
\hline 1 & $\leq(2 n-5)[13,[14]$ & $\leq(n-1)[13,[14]$ & & \\
\hline 2 & $\leq\left\lfloor\frac{3 n-6}{2}\right\rfloor[7$ & & & \\
\hline 3 & $\leq\left\lfloor\frac{22 n-42}{15}\right\rfloor[10]$ & & $\leq(n-1)[\underline{8}]$ & \\
\hline 4 & & $\leq\left\lfloor\frac{5 n}{6}\right\rfloor[18]$ & & $\leq\left\lceil\frac{3 n}{4}\right\rceil[16]$ \\
\hline 5 & $\leq\left\lfloor\frac{13 n-24}{9}\right\rfloor[17]$ & & & \\
\hline 6 & & $\leq\left\lfloor\frac{4 n-1}{5}\right\rfloor[15]$ & & \\
\hline 7 & & $\leq \frac{2 n}{3}+\lfloor 2 \sqrt{n}\rfloor[\underline{6}]$ & & \\
\hline 8 & & $\leq \frac{2 n}{3}+14[\underline{19}]$ & & \\
\hline 9 & $\leq\left\lfloor\frac{4 n}{3}\right\rfloor-2[4]$ & & & $\leq\left\lceil\frac{n}{2}\right\rceil+2\left\lceil\sqrt{\frac{n-2}{2}}\right\rceil[]$ \\
\hline 10 & & & $\leq \frac{3}{2} n[5]$ & $\leq \frac{3}{4} n+2\lceil\sqrt{n}\rceil+4[5]$ \\
\hline \hline
\end{tabular}

Table 1: Previous results on the height and the width of VR.

\section{Introduction}

Drawing plane graphs has emerged as a fast growing research area in recent years (see [3] for a survey). A visibility representation (VR for short) is a classical drawing style of plane graphs where the vertices of a graph $G$ are represented by horizontal line segments (called vertex segment), and each edge of $G$ is represented by a vertical line segment (called edge segment) touching the vertex segments of its end vertices. Figure 1 shows a VR of a plane graph $G$. The problem of finding a VR on a compact grid is important not only in algorithmic graph theory, but also in practical applications. A simple lineartime VR algorithm was given by 13, 14, for 2-connected plane graphs. It uses an st-orientation of $G$ and the corresponding st-orientation of its st-dual $G^{*}$ to construct a VR. Using this approach, the height of the VR is bounded by $(n-1)$ (which is the number of vertices of $G$ minus 1 ) and the width of the VR is bounded by $(2 n-5)$ (which is the number of faces of $G$ minus 1) [13, 14.

As in many other graph drawing problems, one of the main concerns in VR research is to minimize the grid size (i.e., the height and the width) of the representation. For the lower bounds, Zhang and He [18] showed that there exists a plane graph $G$ with $n$ vertices where any VR of $G$ requires a grid of size at least $\left(\left\lfloor\frac{2 n}{3}\right\rfloor\right) \times\left(\left\lfloor\frac{4 n}{3}\right\rfloor-3\right)$. Some works have been done to reduce the height and width of the VR by carefully constructing special st-orientations. Table 1 summarizes related previous results.

Line 1 in Table 1 gives the trivial upper bounds. All other results, except for Line 10, concentrated on one dimension of the VR (either the width or the height). In Table 1 the un-mentioned dimension is bounded by the trivial upper bound, namely, $n-1$ for the height and $2 n-5$ for the width. (For Line 8 , the original bound given in [19] was Height $\leq 2 n / 3+O(1)$. By a more careful calculation, the term $O(1)$ is actually 14.) In [11, 12, heuristic algorithms were developed aiming at reducing the height and the width of VRs at the 
same time. Line 10 in Table 1 is the only VR construction with simultaneously reduced height and width. However, it only works for 4-connected plane graphs.

In this paper, we prove that every plane graph with $n$ vertices has a VR with height at most $\frac{23}{24} n+2\lceil\sqrt{n}\rceil+10$ and width at most $\frac{23}{12} n$.

The present paper is organized as follows. Section2 introduces preliminaries. Section 3 presents a decomposition lemma for plane graphs. Section 4 presents the construction of VR with the stated height and width. Section 5 concludes the paper.

\section{Preliminaries}

In this section, we give definitions and preliminary results. Definitions not mentioned here are standard.

All graphs considered in this paper are simple graphs (namely without selfloops and multiple edges). For a graph $G=(V, E)$ and a subset $U \subset V, G-U$ denotes the graph obtained from $G$ by deleting the vertices in $U$ (and all edges incident to these vertices). A planar graph is a graph $G=(V, E)$ such that the vertices of $G$ can be drawn in the plane and the edges of $G$ can be drawn as non-intersecting curves. Such a drawing is called an embedding. The embedding divides the plane into a number of connected regions. Each region is called a face. The unbounded face is the exterior face. The other faces are interior faces. The vertices and edges that are not on the boundary of the exterior face are called interior vertices and edges, respectively. A plane graph is a planar graph with a fixed embedding. A plane triangulation is a plane graph where every face is a triangle (including the exterior face). We denote the number of vertices of $G$ by $|G|$. The set of interior vertices of $G$ is denoted by $I(G)$. Thus $|I(G)|=|G|-3$ for a plane triangulation $G$.

For a path $P$, length $(P)$ (or $|P|$ ) denotes the number of edges in $P$. For two vertices $a, b$ in $P, P(a, b)$ denotes the sub-path of $P$ from $a$ to $b$ (including $a$ and $b$ ).

When discussing VRs, we assume that, without loss of generality, the input graph $G$ is a plane triangulation. (If not, we add dummy edges into the faces of $G$ to obtain a triangulation $G^{\prime}$. After constructing a VR for $G^{\prime}$, we can get a VR of $G$ by deleting the vertical line segments for the dummy edges). From now on, $G$ always denotes a plane triangulation.

A numbering $\mathcal{O}$ of a set $S=\left\{a_{1}, \ldots, a_{k}\right\}$ is an one-to-one mapping between $S$ and the set $\{1,2, \ldots, k\}$. We write $\mathcal{O}=\left\langle a_{i_{1}}, a_{i_{2}}, \ldots, a_{i_{k}}\right\rangle$ to indicate $\mathcal{O}\left(a_{i_{1}}\right)=1$, $\mathcal{O}\left(a_{i_{2}}\right)=2$, etc. A set $S$ with a numbering written this way is called an ordered list. For two elements $a_{i}$ and $a_{j}$, if $\mathcal{O}\left(a_{i}\right)<\mathcal{O}\left(a_{j}\right)$, we write $a_{i} \prec_{\mathcal{O}} a_{j}$. Let $S_{1}$ and $S_{2}$ be two disjoint sets. If $\mathcal{O}_{1}$ is a numbering of $S_{1}$ and $\mathcal{O}_{2}$ is a numbering of $S_{2}$, their concatenation, written as $\mathcal{O}=\left\langle\mathcal{O}_{1}, \mathcal{O}_{2}\right\rangle$, is the numbering of $S_{1} \cup S_{2}$ defined by:

$$
\mathcal{O}(x)= \begin{cases}\mathcal{O}_{1}(x) & \text { for all } x \in S_{1} \\ \mathcal{O}_{2}(x)+\left|S_{1}\right| & \text { for all } x \in S_{2}\end{cases}
$$


$G$ is called a directed graph (digraph for short) if each edge of $G$ is assigned a direction. An orientation of an undirected graph $G$ is a digraph obtained from $G$ by assigning a direction to each edge of $G$. We use $G$ to denote both the resulting digraph and the underlying undirected graph unless otherwise specified. (Its meaning will be clear from the context.)

Let $G=(V, E)$ be an undirected graph. A numbering $\mathcal{O}$ of $V$ induces an orientation of $G$ as follows: each edge $(u, v)$ of $G$ is directed from $u$ to $v$ if and only if $\mathcal{O}(u)<\mathcal{O}(v)$. The resulting digraph, denoted by $G_{\mathcal{O}}$, is called the orientation derived from $\mathcal{O}$ which, obviously, is an acyclic digraph. We use length $_{G}(\mathcal{O})$ (or simply length $(\mathcal{O})$ if $G$ is clear from the context) to denote the length of the longest directed path in $G_{\mathcal{O}}$.

For a 2-connected plane graph $G$ and an exterior edge $(s, t)$, an orientation of $G$ is called an st-orientation if the following conditions hold:

- the resulting digraph is acyclic;

- $s$ is the only source and $t$ is the only sink.

Such a digraph is also called an st-graph. Lempel et al. 9 showed that for every 2-connected plane graph $G$ and an exterior edge $(s, t)$, there exists an $s t$-orientation. The properties of $s t$-orientations and $s t$-graphs can be found in [2].

Let $G$ be a 2-connected plane graph and $(s, t)$ an exterior edge. An stnumbering of $G$ is an one-to-one mapping $\xi: V \rightarrow\{1,2, \ldots, n\}$ such that $\xi(s)=1, \xi(t)=n$, and each vertex $v \neq s, t$ has two neighbors $u, w$ with $\xi(u)<\xi(v)<\xi(w)$, where $u$ ( $w$, resp.) is called a smaller neighbor (bigger neighbor, resp.) of $v$. Given an st-numbering $\xi$ of $G$, the orientation of $G$ derived from $\xi$ is obviously an $s t$-orientation of $G$. On the other hand, if $G=(V, E)$ has an st-orientation $\mathcal{O}$, we can define an one-to-one mapping $\xi: V \rightarrow\{1, \ldots, n\}$ by topological sort. It is easy to see that $\xi$ is an st-numbering and the orientation derived from $\xi$ is $\mathcal{O}$. From now on, we will interchangeably use the term "an st-numbering" of $G$ and the term "an st-orientation" of $G$, where each edge of $G$ is directed accordingly.

Definition 1 Let $G$ be a plane graph with an st-orientation $\mathcal{O}$, where $(s, t)$ is an exterior edge drawn at the left on the exterior face of $G$. The st-dual $G^{*}$ of $G$ and the dual orientation $\mathcal{O}^{*}$ of $\mathcal{O}$ are defined as follows:

- Each face $f$ of $G$ corresponds to a node $f^{*}$ of $G^{*}$. In particular, the unique interior face adjacent to the edge $(s, t)$ corresponds to a node $s^{*}$ in $G^{*}$, the exterior face corresponds to a node $t^{*}$ in $G^{*}$.

- For each edge $e \neq(s, t)$ of $G$ separating a face $f_{1}$ on its left and a face $f_{2}$ on its right, there is a dual edge $e^{*}$ in $G^{*}$ from $f_{1}^{*}$ to $f_{2}^{*}$.

- The dual edge of the exterior edge $(s, t)$ is directed from $s^{*}$ to $t^{*}$.

Figure1(a) shows an st-graph $G$ and its $s t$-dual graph $G^{*}$. (Circles and solid lines denote the vertices and the edges of $G$. Squares and dashed lines denote the 

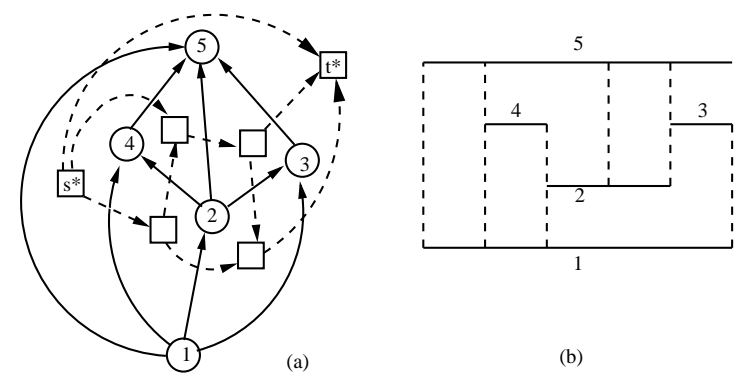

(b)

Figure 1: (a) An st-graph $G$ and its $s t$-dual graph $G^{*}$; (b) A VR of $G$.

nodes and the edges of $G^{*}$.) It is well known that the st-dual graph $G^{*}$ defined above is an $s t$-graph with source $s^{*}$ and sink $t^{*}, 2,13,14$. The correspondence between an st-orientation $\mathcal{O}$ of $G$ and the dual st-orientation $\mathcal{O}^{*}$ is an one-to-one correspondence. The following theorem was proved in [13, 14]:

Theorem 1 Let $G$ be a 2-connected plane graph with an st-orientation $\mathcal{O}$. Let $\mathcal{O}^{*}$ be the dual st-orientation of $G^{*} . A V R$ of $G$ can be obtained from $\mathcal{O}$ and $\mathcal{O}^{*}$ in linear time. The height of the $V R$ is $\operatorname{length}(\mathcal{O})$. The width of the VR is length $\left(\mathcal{O}^{*}\right)$. Since $G$ has $n$ vertices and $G^{*}$ has $2 n-4$ nodes, any st-orientation of $G$ leads to a VR with height at most $n-1$ and width at most $2 n-5$.

Figure 1 (b) shows a VR of the graph $G$ shown in Figure 1 (a). The width of the $\mathrm{VR}$ is length $\left(\mathcal{O}^{*}\right)=5$. The height of the $\mathrm{VR}$ is length $(\mathcal{O})=3$.

The following theorems were given in [19, 7, 5, and will be needed later for our VR construction.

Theorem 2 [19] Every plane triangulation with $n$ vertices has a VR with width at most $2 n-5$ and height at most $\frac{2}{3} n+14$, which can be constructed in linear time.

Theorem 3 [7] Every plane triangulation with n vertices has a VR with height at most $n-1$ and width at most $\left\lfloor\frac{3 n-6}{2}\right\rfloor$, which can be constructed in linear time and we can specify $s$ and $t$ arbitrarily on the exterior face.

Theorem 4 [5] Every 4-connected plane triangulation with $n$ vertices has a $V R$ with height at most $\frac{3}{4} n+2\lceil\sqrt{n}\rceil+4$ and width at most $\frac{3}{2} n$, which can be constructed in linear time.

Due to Theorem 1 the results in the above theorems can also be stated in terms of the lengths of the orientations of $G$. The statement " $G$ has an storientation $\mathcal{O}$ such that length $(\mathcal{O}) \leq x$ and $\operatorname{length}\left(\mathcal{O}^{*}\right) \leq y$ " is equivalent to the statement "the VR of $G$ derived from $\mathcal{O}$ has height at most $x$ and width at most $y$ ". We will use these two statements interchangeably. 


\section{A Decomposition Lemma}

The basic idea of our VR construction is as follows. First, we divide the input graph $G$ into several subgraphs. Then we use the VR constructions in Theorems 1, 2, 3 and 4 for different subgraphs of $G$. Some of them have small width and others have small height. The main difficulty of our VR construction is to find a proper balance on the sizes of these subgraphs so that the overall height and width of the VR are both reduced. In this section, we prove a decomposition lemma that is needed by our VR construction to achieve the balance.

Let $G=(V, E)$ be a plane graph. A triangle of $G$ is a set of three mutually adjacent vertices. The notation $\triangle=\{a, b, c\}$ denotes a triangle consisting of vertices $a, b, c$. A triangle divides the plane into an interior region and an exterior region. We say that $\triangle=\{a, b, c\}$ is a separating triangle if $G-\{a, b, c\}$ is disconnected. In other words, $\triangle$ is a separating triangle if both its interior and exterior regions contain vertices. The following fact by Whitney is well known,

Fact 1 A plane triangulation $G$ is 4-connected if and only if $G$ has no separating triangles.

Let $\triangle=\{a, b, c\}$ be a separating triangle. Then $G_{\triangle}$ denotes the subgraph of $G$ induced by $\{a, b, c\} \cup\{v \in V \mid v$ is in the interior of $\triangle\}$. We say that $\triangle$ is maximal if there is no other separating triangle $\triangle^{\prime}$ such $G_{\triangle} \subset G_{\triangle^{\prime}}$. Two triangles $\triangle_{1}$ and $\triangle_{2}$ are related if either $G_{\triangle_{1}} \subseteq G_{\triangle_{2}}$ or $G_{\triangle_{2}} \subseteq G_{\triangle_{1}}$.

Let $G_{1}$ and $G_{2}$ be two plane triangulations. If $G_{1}$ has an internal face $f$ such that the vertex set of $f$ and the vertex set of the outer face of $G_{2}$ are identical, we can embedded $G_{2}$ into $G_{1}$ by identifying the face $f$ and the exterior face of $G_{2}$. The resulting plane triangulation is denoted by $G_{1} \oplus_{f} G_{2}$ (or simply $\left.G_{1} \oplus G_{2}\right)$.

Definition 2 Let $G_{1}$ and $G_{2}$ be two plane triangulations such that $G_{2}$ can be embedded into $G_{1}$ by a common face $f=\{a, b, c\}$. Let $\mathcal{O}_{1}$ be an storientation of $G_{1}$ and let $\mathcal{O}_{2}$ be an st-orientation of $G_{2}$ such that the three edges $\{(a, b),(b, c),(c, a)\}$ are oriented the same way in $\mathcal{O}_{1}$ and $\mathcal{O}_{2} . \mathcal{O}_{G_{1}} \oplus \mathcal{O}_{G_{2}}$ denotes the union of $\mathcal{O}_{1}$ and $\mathcal{O}_{2}$, which is an orientation of $G_{1} \oplus G_{2}$.

Lemma 1 Let $G_{1}, G_{2}, \mathcal{O}_{1}$, and $\mathcal{O}_{2}$ be as in Definition 2. Then $\mathcal{O}_{G_{1}} \oplus \mathcal{O}_{G_{2}}$ is an st-orientation of $G_{1} \oplus G_{2}$.

Proof: Immediate from the definition.

Definition 3 The 4 -block tree of a plane triangulation $G$ is a rooted tree $T$ defined as follows:

- If $G$ has no separating triangles (i.e., $G$ is 4 -connected), then $T$ consists of a single root $r$. 


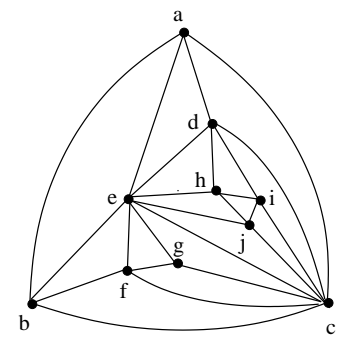

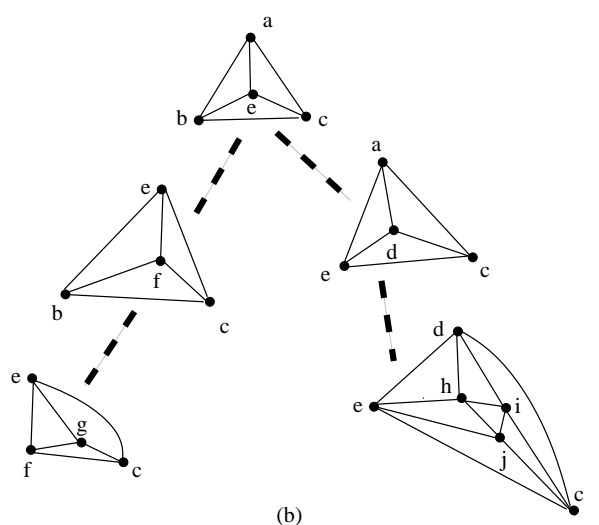

(b)

Figure 2: (a) A triangulation $G$; (b) 4-block components and the 4-block tree $T$ of $G$.

- If not, let $\triangle_{1}, \ldots, \triangle_{p}$ be the maximal separating triangles of $G$. Let $T_{i}$ be the 4-block tree of $G_{\triangle_{i}}$. Then $T$ is the tree with root $r$ and the roots of $T_{i}(1 \leq i \leq p)$ as the children of $r$.

From the definition, we have the following properties:

- Each non-root node $u$ of $T$ corresponds to a separating triangle $\triangle_{u}$ of $G$.

- For any $u, v \in T, u$ and $v$ have ancestor-descendant relation if and only if $\triangle_{u}$ and $\triangle_{v}$ are related in $G$.

For a node $u$ of $T, G_{u}$ denotes the subgraph $G_{\triangle_{u}}-\left(\bigcup_{v \in C(u)} I\left(G_{\triangle_{v}}\right)\right)$ where $C(u)$ is the set of children of $u$ in $T$. In other words, $G_{u}$ is obtained from $G_{\triangle_{u}}$ by deleting all vertices that are in the interior of the maximal separating triangles of $G_{\triangle_{u}}$. Since $G_{u}$ has no separating triangles, $G_{u}$ is 4-connected. Each $G_{u}$ is called a 4-block component of $G$. Figure 2 shows a plane triangulation $G$, the 4-block components and the 4-block tree of $G$. For a node $u \in T$, for convenience, we use $\left|T_{u}\right|$ to denote $\left|G_{\triangle_{u}}\right|$.

For example, consider the graph $G$ and its decomposition tree $T$ shown in Figure 2. Let $u$ be the node of $T$ that is the right child of the root of $T$ (consisting of the vertices $\{a, c, d, e\}$.) Then the graph $G_{u}$ consists of the four vertices $\{a, c, d, e\} . G_{\triangle_{u}}$ is the subgraph of $G$ consisting of the vertices $a, e, c$ and all vertices contained in the interior region of the separating triangle $\triangle=\{a, e, c\}$. We have $\left|T_{u}\right|=7$.

Lemma 2 Let $G$ be a triangulation and $T$ be its 4-block tree. Then at least one of the following two conditions holds.

1. There exists a node $v$ in $T$ such that $\left|G_{v}\right| \geq \frac{n}{6}$. 
2. There exists a set of unrelated separating triangles $\left\{\triangle_{1}, \triangle_{2}, \ldots, \triangle_{h}\right\}$ such that $\left|G_{\triangle_{i}}\right| \geq 5$ and $\frac{n}{4}-3 \leq \sum_{i=1}^{h}\left|I\left(G_{\triangle_{i}}\right)\right| \leq \frac{3}{4} n-3$.

Moreover, the decomposition can be found in linear time.

Proof: Let $r$ be the root of $T$. Let $H$ be a maximal path in $T$ from $r$ to some node $v$ of $T$ such that for each node $u \in H,\left|T_{u}\right| \geq \frac{3 n}{4}$ ( $v$ can be the root $r$ ).

If $v$ is a leaf of $T$, then $\left|G_{v}\right| \geq \frac{3 n}{4}>\frac{n}{6}$. So condition (1) is satisfied.

Now, suppose $v$ is not a leaf. Let $\left\{v_{1}, v_{2}, \ldots, v_{p}\right\}$ be the children of $v$ in $T$. Re-arrange the indices, if necessary, so that $\left|T_{v_{1}}\right| \leq\left|T_{v_{2}}\right| \leq \ldots \leq\left|T_{v_{p}}\right|$. Then, either $\frac{n}{4} \leq\left|T_{v_{p}}\right|<\frac{3 n}{4}$; or $\left|T_{v_{i}}\right|<\frac{n}{4}$ for all $v_{i} \in\left\{v_{1}, v_{2}, \ldots, v_{p}\right\}$.

Case A: suppose $\left|T_{v_{i}}\right|<\frac{n}{4}$ for all $v_{i}$. Let $i^{*} \in\{1, \cdots, p\}$ be the index such that $\left|T_{v_{i}}\right| \leq 4$ for all $i \leq i^{*}$ and $\left|T_{v_{i}}\right| \geq 5$ for all $i>i^{*}$. There are three sub-cases.

1. $\sum_{i>i^{*}}\left(\left|T_{v_{i}}\right|-3\right)<\frac{n}{4}-3$.

Let $n_{1}=\left|G_{v}\right|$. Since $G_{v}$ is a triangulation with $n_{1}$ vertices, $G_{v}$ has $2 n_{1}-5$ internal faces by Euler's formula. Each child $v_{i}$ of $v$ corresponds to a maximal separating triangle of $G_{\triangle_{v}}$, and each such separating triangle is one of the interior faces of $G_{v}$. Thus, $i^{*} \leq p \leq 2 n_{1}-5$. Since $\left|I\left(G_{\triangle_{v_{i}}}\right)\right|=1$ for all $i \leq i^{*}$, we have

$$
\begin{aligned}
\frac{3}{4} n & \leq\left|T_{v}\right|=n_{1}+\sum_{i \leq i^{*}}\left|I\left(G_{\triangle_{v_{i}}}\right)\right|+\sum_{i>i^{*}}\left|I\left(G_{\triangle_{v_{i}}}\right)\right|=n_{1}+i^{*}+\sum_{i>i^{*}}\left|I\left(G_{\triangle_{v_{i}}}\right)\right| \\
& \leq n_{1}+\left(2 n_{1}-5\right)+\sum_{i>i^{*}}\left|I\left(G_{\triangle_{v_{i}}}\right)\right|
\end{aligned}
$$

From the assumption $\sum_{i>i^{*}}\left|I\left(G_{\triangle_{v_{i}}}\right)\right|<\frac{n}{4}-3$, we have: $3 n_{1}-5>$ $\frac{3}{4} n-\frac{n}{4}+3=\frac{n}{2}+3$. This implies $\left|G_{v}\right|=n_{1} \geq \frac{n}{6}+\frac{8}{3}$. So $G_{v}$ satisfies condition (1).

2. $\frac{n}{4}-3 \leq \sum_{i>i^{*}}\left(\left|T_{v_{i}}\right|-3\right) \leq \frac{3}{4} n-3$

This is equivalent to $\frac{n}{4}-3 \leq \sum_{i>i^{*}}\left|I\left(G_{\triangle_{v_{i}}}\right)\right| \leq \frac{3}{4} n-3$. So the set of unrelated separating triangles $\left\{\triangle_{v_{i^{*}+1}}, \triangle_{v_{i^{*}+2}}, \ldots, \triangle_{v_{i_{p}}}\right\}$ satisfies condition (2).

3. $\sum_{i>i^{*}}\left(\left|T_{v_{i}}\right|-3\right)>\frac{3}{4} n-3$

Let $i_{t}$ be the first index such that $\sum_{i^{*}<i \leq i_{t}}\left(\left|T_{v_{i}}\right|-3\right) \geq \frac{n}{4}-3$. Because $\left|T_{v_{i}}\right|<\frac{n}{4}$ for each $i$, clearly $\sum_{i^{*}<i \leq i_{t}}\left(\left|T_{v_{i}}\right|-3\right) \leq \frac{3}{4} n-3$. So the set of unrelated separating triangles $\left\{\triangle_{v_{i^{*}+1}}, \triangle_{v_{i^{*}+2}}, \ldots, \triangle_{v_{i_{t}}}\right\}$ satisfies condition (2).

Case B: $\frac{n}{4} \leq\left|T_{v_{p}}\right|<\frac{3 n}{4}$. If $\left|T_{v_{p}}\right|>4$, then the separating triangle $\triangle_{v_{p}}$ satisfies $\frac{n}{4}-3 \leq\left|I\left(G_{\triangle_{v_{p}}}\right)\right| \leq \frac{3 n}{4}-3$. So the single separating triangle $\triangle_{v_{p}}$ satisfies condition (2).

Otherwise, $\left|T_{v_{p}}\right| \leq 4$. This is a special case of Case A (1) (where $i^{*}=p$ ). So the claim holds. 
For the run time, we first construct the 4-block tree and the 4-block components of $G$. This can be done in linear time [7. The sizes of the 4-block components can be easily calculated in linear time. Since the decomposition is solely determined by the sizes of these 4-block components, it can also be done in linear time.

\section{Compact Visibility Representation}

In this section, we describe our compact VR construction of a plane triangulation $G$. In order to keep the VR's height and width small simultaneously, we construct a VR of $G$ by using different VRs for some subgraphs of $G$. As stated in Theorems 1 2, 3) and 4, some of these VRs have small height and others have small width. Roughly speaking, we select a set of unrelated separating triangles $\left\{\triangle_{1}, \triangle_{2}, \ldots, \triangle_{h}\right\}$ of $G$. Let $G^{\prime}$ be the subgraph of $G$ consisting of the vertices that are outside of $\left\{G_{\triangle_{1}}, G_{\triangle_{2}}, \ldots, G_{\triangle_{h}}\right\}$. We use a VR of $G^{\prime}$ with small height. For each $G_{\triangle_{i}}$, we use a VR with small width. Then, we embed each $G_{\triangle_{i}}$ into $G^{\prime}$.

For convenience, define the function $\mathcal{X}(k)=\left\lceil\frac{k}{2}-\frac{1}{2}\right\rceil$ for integers $k \geq 1$. It is easy to verify that

- $\mathcal{X}(k)$ is non-decreasing .

- $\mathcal{X}(k) \geq 1$ and $\mathcal{X}(k) \geq k / 3$ for all $k \geq 2$.

Theorem 5 Let $S=\left\{\triangle_{1}, \triangle_{2}, \ldots, \triangle_{h}\right\}$ be a set of unrelated separating triangles of $G$. Then $G$ has an st-orientation $\mathcal{O}$ such that length $(\mathcal{O}) \leq \frac{2 n}{3}+$ $\frac{\sum_{i=1}^{h}\left|I\left(G_{\triangle_{i}}\right)\right|}{3}+14$ and length $\left(\mathcal{O}^{*}\right) \leq 2 n-5-\sum_{i=1}^{h} \mathcal{X}\left(\left|I\left(G_{\triangle_{i}}\right)\right|\right)$.

Proof: Define $G_{j}=G-\bigcup_{i=j+1}^{h} I\left(G_{\triangle_{i}}\right)$. (In other words, $G_{j}$ is obtained from $G$ by deleting all vertices in the interior of the separating triangles $\triangle_{i}$ for $j+1 \leq i \leq h$.) Note that $G=G_{h}$.

We will show that $G_{j}(0 \leq j \leq h)$ has an st-orientation $\mathcal{O}_{j}$ so that

Claim 1 length $\left(\mathcal{O}_{j}\right) \leq \frac{2}{3}\left|G_{j}\right|+14+\frac{1}{3} \sum_{i=1}^{j}\left|I\left(G_{\triangle_{i}}\right)\right|$.

Claim 2 length $\left(\mathcal{O}_{j}^{*}\right) \leq 2\left|G_{j}\right|-5-\sum_{i=1}^{j} \mathcal{X}\left(\left|I\left(G_{\triangle_{i}}\right)\right|\right)$.

Then the theorem follows. We prove these claims by induction.

Base case $j=0$ : From Theorem $2 G_{0}$ has an st-orientation $\mathcal{O}_{0}$ such that $\operatorname{length}\left(\mathcal{O}_{0}\right) \leq \frac{2}{3}\left|G_{0}\right|+14$ and length $\left(\mathcal{O}_{0}^{*}\right) \leq 2\left|G_{0}\right|-5$. So the claims hold for the base case.

Induction hypothesis: $G_{k}$ has an st-orientation $\mathcal{O}_{k}$ such that

$$
\operatorname{length}\left(\mathcal{O}_{k}\right) \leq \frac{2}{3}\left|G_{k}\right|+14+\frac{1}{3} \sum_{i=1}^{k}\left|I\left(G_{\triangle_{i}}\right)\right|
$$




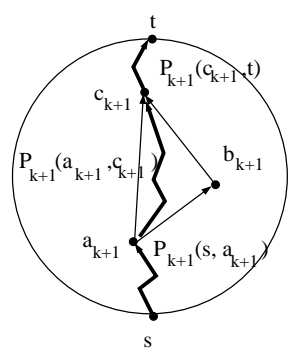

(a)

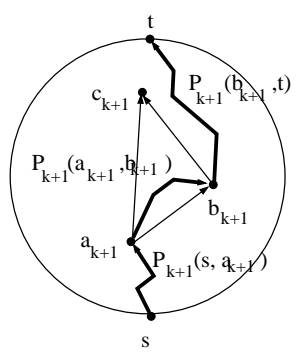

(b)

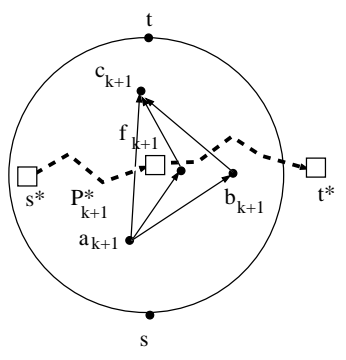

(c)

Figure 3: The proof of Theorem 5 (a) Case (ii); (b) Case (iii); (c) Path in the dual graph.

and

$$
\operatorname{length}\left(\mathcal{O}_{k}^{*}\right) \leq 2\left|G_{k}\right|-5-\sum_{i=1}^{k} \mathcal{X}\left(\left|I\left(G_{\triangle_{i}}\right)\right|\right) .
$$

Suppose that $\triangle_{k+1}=\left\{a_{k+1}, b_{k+1}, c_{k+1}\right\}$. Without loss of generality, assume that the edges of $\triangle_{k+1}$ are oriented in $\mathcal{O}_{k}$ as $\left(a_{k+1} \rightarrow b_{k+1}\right),\left(b_{k+1} \rightarrow\right.$ $\left.c_{k+1}\right),\left(a_{k+1} \rightarrow c_{k+1}\right)$.

By Theorem 3, $G_{\triangle_{k+1}}$ has an st-orientation $\mathcal{O}_{\triangle_{k+1}}$ from $a_{k+1}$ to $c_{k+1}$ such that length $\left(\mathcal{O}_{\triangle_{k+1}}\right) \leq\left|G_{\triangle_{k+1}}\right|-1$ and length $\left(\mathcal{O}_{\triangle_{k+1}}^{*}\right) \leq\left\lfloor\frac{3\left|G_{\triangle_{k+1}}\right|-6}{2}\right\rfloor$.

Let $\mathcal{O}_{k+1}=\mathcal{O}_{k} \oplus \mathcal{O}_{\triangle_{k+1}}$.

First we show length $\left(\mathcal{O}_{k+1}\right) \leq \frac{2}{3}\left|G_{k+1}\right|+14+\frac{1}{3} \sum_{i=1}^{k+1}\left|I\left(G_{\triangle_{i}}\right)\right|$.

Note that $\left|G_{k+1}\right|=\left|G_{k}\right|+\left|I\left(G_{\triangle_{k+1}}\right)\right|=\left|G_{k}\right|+\left|G_{\triangle_{k+1}}\right|-3$.

Let $P_{k+1}$ be a longest path in $\mathcal{O}_{k+1}$ from $s$ to $t$ in $G_{k+1}$; let $P_{k}$ be a longest path in $\mathcal{O}_{k}$ from $s$ to $t$ in $G_{k}$; and let $P_{\triangle_{k+1}}$ be a longest path in $\mathcal{O}_{\triangle_{k+1}}$ from $a_{k+1}$ to $c_{k+1}$. There are several cases:

$P_{k+1}$ does not contain any interior edge in $G_{\triangle_{k+1}}$. Then $P_{k+1}$ is a path in $G_{k}$. By induction hypothesis,

$$
\begin{aligned}
\operatorname{length}\left(\mathcal{O}_{k+1}\right)=\left|P_{k+1}\right| & \leq \frac{2}{3}\left|G_{k}\right|+14+\frac{1}{3} \sum_{i=1}^{k}\left|I\left(G_{\triangle_{i}}\right)\right| \\
& <\frac{2}{3}\left|G_{k+1}\right|+14+\frac{1}{3} \sum_{i=1}^{k+1}\left|I\left(G_{\triangle_{i}}\right)\right|
\end{aligned}
$$

(ii) $P_{k+1}$ passes through a path in $G_{\triangle_{k+1}}$ from $a_{k+1}$ to $c_{k+1}$ (see Figure[3)(a)). $P_{k+1}$ can be divided into three sub-paths: $P_{k+1}\left(s, a_{k+1}\right), P_{k+1}\left(a_{k+1}, c_{k+1}\right)$, $P_{k+1}\left(c_{k+1}, t\right)$. Here $P_{k+1}\left(s, a_{k+1}\right), P_{k+1}\left(c_{k+1}, t\right)$ are paths in $G_{k}$, while $P_{k+1}\left(a_{k+1}, c_{k+1}\right)$ is a path in $G_{\triangle_{k+1}}$. Since $P_{\triangle_{k+1}}$ is a longest path in $G_{\triangle_{k+1}}$, we have $\left|P_{k+1}\left(a_{k+1}, c_{k+1}\right)\right| \leq\left|P_{\triangle_{k+1}}\right|$.

Let $P^{\prime}$ be the concatenation of $P_{k+1}\left(s, a_{k+1}\right)$ followed by the edges $\left(a_{k+1} \rightarrow\right.$ $\left.b_{k+1}\right)$ and $\left(b_{k+1} \rightarrow c_{k+1}\right)$; followed by $P_{k+1}\left(c_{k+1}, t\right)$. Then $P^{\prime}$ is a path in 
$G_{k}$. Thus $\left|P^{\prime}\right|=\left|P_{k+1}\left(s, a_{k+1}\right)\right|+2+\left|P_{k+1}\left(c_{k+1}, t\right)\right| \leq\left|P_{k}\right|$. This implies $\left|P_{k+1}\left(s, a_{k+1}\right)\right|+\left|P_{k+1}\left(c_{k+1}, t\right)\right| \leq\left|P_{k}\right|-2$. Hence

$$
\begin{aligned}
\operatorname{length}\left(\mathcal{O}_{k+1}\right) & =\left|P_{k+1}\right|=\left|P_{k+1}\left(s, a_{k+1}\right)\right|+\left|P_{k+1}\left(a_{k+1}, c_{k+1}\right)\right|+\left|P_{k+1}\left(c_{k+1}, t\right)\right| \\
& \leq\left|P_{k}\right|-2+\left|P_{\triangle_{k+1}}\right| \\
& \leq \frac{2}{3}\left|G_{k}\right|+14+\frac{1}{3} \sum_{i=1}^{k}\left|I\left(G_{\triangle_{i}}\right)\right|-2+\left|G_{\triangle_{k+1}}\right|-1 \\
& =\frac{2}{3}\left|G_{k}\right|+\frac{1}{3} \sum_{i=1}^{k}\left|I\left(G_{\triangle_{i}}\right)\right|+\left[\left|I\left(G_{\triangle_{k+1}}\right)\right|+3\right]+14-3 \\
& =\frac{2}{3}\left(\left|G_{k}\right|+\left|I\left(G_{\triangle_{k+1}}\right)\right|\right)+\frac{1}{3} \sum_{i=1}^{k+1}\left|I\left(G_{\triangle_{i}}\right)\right|+14 \\
& =\frac{2}{3}\left|G_{k+1}\right|+14+\frac{1}{3} \sum_{i=1}^{k+1}\left|I\left(G_{\triangle_{i}}\right)\right|
\end{aligned}
$$

(iii) $P_{k+1}$ passes through a path in $G_{\triangle_{k+1}}$ from $a_{k+1}$ to $b_{k+1}$ (see Figure 3 (b)).

$P_{k+1}$ can be divided into three sub-paths $P_{k+1}\left(s, a_{k+1}\right), P_{k+1}\left(a_{k+1}, b_{k+1}\right)$, $P_{k+1}\left(b_{k+1}, t\right)$. Here $P_{k+1}\left(s, a_{k+1}\right)$ and $P_{k+1}\left(b_{k+1}, t\right)$ are paths in $G_{k}$, while $P_{k+1}\left(a_{k+1}, b_{k+1}\right)$ is a path in $G_{\triangle_{k+1}}$. The concatenation of $P_{k+1}\left(a_{k+1}, b_{k+1}\right)$ and the edge $b_{k+1} \rightarrow c_{k+1}$ is a path in $G_{\triangle_{k+1}}$. Hence $\left|P_{k+1}\left(a_{k+1}, b_{k+1}\right)\right|+1 \leq$ $\left|P_{\triangle_{k+1}}\right|$.

The concatenation of $P_{k+1}\left(s, a_{k+1}\right)$ followed by the edge $a_{k+1} \rightarrow b_{k+1}$, followed by $P_{k+1}\left(b_{k+1}, t\right)$ is a path in $G_{k}$. So we have that $\left|P_{k+1}\left(s, a_{k+1}\right)\right|+1+$ $\left|P_{k+1}\left(b_{k+1}, t\right)\right| \leq\left|P_{k}\right|$. Hence

$$
\begin{aligned}
\operatorname{length}\left(\mathcal{O}_{k+1}\right) & =\left|P_{k+1}\right|=\left|P_{k+1}\left(s, a_{k+1}\right)\right|+\left|P_{k+1}\left(a_{k+1}, b_{k+1}\right)\right|+\left|P_{k+1}\left(b_{k+1}, t\right)\right| \\
& \leq\left(\left|P_{k}\right|-1\right)+\left(\left|P_{\triangle_{k+1}}\right|-1\right) \\
& =\left|P_{k}\right|-2+\left|P_{\triangle_{k+1}}\right| \\
& \leq \frac{2}{3}\left|G_{k+1}\right|+14+\frac{1}{3} \sum_{i=1}^{k+1}\left|I\left(G_{\triangle_{i}}\right)\right| .
\end{aligned}
$$

The proof of the last inequality is the same as the proof of case (ii).

(iv) $P_{k+1}$ passes through a path in $G_{\triangle_{k+1}}$ from $b_{k+1}$ to $c_{k+1}$. The proof is symmetric to case (iii).

Next we prove Claim 2. Let $P_{k}^{*}$ be a longest path from $s^{*}$ to $t^{*}$ in $\mathcal{O}_{k}^{*}$. From the induction hypothesis, we know that $\left|P_{k}^{*}\right| \leq 2\left|G_{k}\right|-5-\sum_{i=1}^{k} \mathcal{X}\left(\left|I\left(G_{\triangle_{i}}\right)\right|\right)$. Let $P_{\triangle_{k+1}}^{*}$ be a longest path in $G_{\triangle_{k+1}}^{*}$. By Theorem [3, $\left|P_{\triangle_{k+1}}^{*}\right| \leq\left\lfloor\frac{3\left|G_{\triangle_{k+1}}\right|-6}{2}\right\rfloor$. 
Let $P_{k+1}^{*}$ be a longest path from $s^{*}$ to $t^{*}$ in $\mathcal{O}_{k+1}^{*}$. Let $f_{k+1}$ be the face in $G_{k+1}$ that is in the interior of $\triangle_{k+1}$ adjacent to the edge $a_{k+1} \rightarrow c_{k+1}$ (see Figure 3 (c).) (In other words, $f_{k+1}$ corresponds to the source node of the dual st-orientation of $G_{\triangle_{k+1}}^{*}$.) If $P_{k+1}^{*}$ uses any edge in $G_{\triangle_{k+1}}^{*}$, it must cross the edge $a_{k+1} \rightarrow c_{k+1}$ and enter the face $f_{k+1}$. There are two cases.

(a) $P_{k+1}^{*}$ does not go through $f_{k+1}$. Then $P_{k+1}^{*}$ is a path in $G_{k}^{*}$ and the claim trivially holds:

$$
\begin{aligned}
\left|P_{k+1}^{*}\right| & \leq 2\left|G_{k}\right|-5-\sum_{i=1}^{k} \mathcal{X}\left(\left|I\left(G_{\triangle_{i}}\right)\right|\right) \\
& =2\left(\left|G_{k}\right|+\left|I\left(G_{\triangle_{k+1}}\right)\right|\right)-5-2\left|I\left(G_{\triangle_{k+1}}\right)\right|-\sum_{i=1}^{k} \mathcal{X}\left(\left|I\left(G_{\triangle_{i}}\right)\right|\right) \\
& \leq 2\left|G_{k+1}\right|-5-\sum_{i=1}^{k+1} \mathcal{X}\left(I\left(G_{\triangle_{i}}\right)\right)
\end{aligned}
$$

(b) $P_{k+1}^{*}$ passes through $f_{k+1}$.

$$
\begin{aligned}
\operatorname{length}\left(\mathcal{O}_{k+1}^{*}\right)= & \left|P_{k}^{*}\right|+\left|P_{\triangle_{k+1}}^{*}\right|-\left|\left\{f_{k+1}\right\}\right| \\
\leq & \left.2\left|G_{k}\right|-5-\sum_{i=1}^{k} \mathcal{X}\left(\left|I\left(G_{\triangle_{i}}\right)\right|\right)+\mid \frac{3\left|G_{\triangle_{k+1}}\right|-6}{2}\right\rfloor-1 \\
= & 2\left(\left|G_{k+1}\right|-\left|I\left(G_{\triangle_{k+1}}\right)\right|\right)-5-\sum_{i=1}^{k} \mathcal{X}\left(\left|I\left(G_{\triangle_{i}}\right)\right|\right)+ \\
& +\left|\frac{3\left(\left|I\left(G_{\triangle_{k+1}}\right)\right|+3\right)-6}{2}-1\right| \\
= & 2\left|G_{k+1}\right|-5-\sum_{i=1}^{k} \mathcal{X}\left(\left|I\left(G_{\triangle_{i}}\right)\right|\right)-2\left|I\left(G_{\triangle_{k+1}}\right)\right|+ \\
& \left.+\mid \frac{3\left|I\left(G_{\triangle_{k+1}}\right)\right|}{2}+\frac{1}{2}\right\rfloor \\
= & 2\left|G_{k+1}\right|-5-\sum_{i=1}^{k} \mathcal{X}\left(\left|I\left(G_{\triangle_{i}}\right)\right|\right)-\left(\left[\frac{\mid I\left(G_{\triangle_{k+1}} \mid\right.}{2}-\frac{1}{2} \mid\right)\right. \\
= & 2\left|G_{k+1}\right|-5-\sum_{i=1}^{k+1} \mathcal{X}\left(\left|I\left(G_{\triangle_{i}}\right)\right|\right)
\end{aligned}
$$

This completes the induction.

Lemma 3 Let $S=\left\{\triangle_{1}, \triangle_{2}, \ldots, \triangle_{h}\right\}$ be a set of unrelated separating triangles of $G$ such that $G^{\prime}=G-\left(\bigcup_{i=1}^{h} I\left(G_{\triangle_{i}}\right)\right)$ is a 4-connected graph. Then, $G$ has an 
st-orientation $\mathcal{O}$ such that length $(\mathcal{O}) \leq \frac{3}{4} n+\frac{\sum_{i=1}^{h}\left|I\left(G_{\triangle_{i}}\right)\right|}{4}+2\left\lceil\sqrt{\left|G^{\prime}\right|}\right\rceil+4$ and $\operatorname{length}\left(\mathcal{O}^{*}\right) \leq \frac{3}{2} n+\frac{\sum_{i=1}^{h}\left|I\left(G_{\triangle_{i}}\right)\right|}{2}$.

Proof: The idea of the proof is very similar to the proof of Theorem 5. The only difference is that here the graph $G^{\prime}$ is assumed to be 4-connected. So we can construct an st-orientation of $G^{\prime}$ in Theorem 4. In contrast, in the proof of Theorem [5 without the 4-connectivity assumption, we use the st-orientation in Theorem 2.

Define $G_{j}=G-\bigcup_{i=j+1}^{h} I\left(G_{\triangle_{i}}\right)$. We show, by induction, that $G_{j}$ has an st-orientation $\mathcal{O}_{j}$ such that

Claim 1: length $\left(\mathcal{O}_{j}\right) \leq \frac{3}{4}\left|G_{j}\right|+\frac{\sum_{i=1}^{j}\left|I\left(G_{\triangle_{i}}\right)\right|}{4}+2\left\lceil\sqrt{\left|G^{\prime}\right|}\right\rceil+4$ and

Claim 2: length $\left(\mathcal{O}_{j}^{*}\right) \leq \frac{3}{2}\left|G_{j}\right|+\frac{\sum_{i=1}^{j}\left|I\left(G_{\triangle_{i}}\right)\right|}{2}$.

Base case $j=0$ : Since $G_{0}=G^{\prime}$ is 4 -connected, by Theorem 4, $G^{\prime}$ has an st-orientation $\mathcal{O}^{\prime}$ such that length $\left(\mathcal{O}^{\prime}\right) \leq \frac{3}{4}\left|G^{\prime}\right|+2\left\lceil\sqrt{\left|G^{\prime}\right|}\right\rceil+4$ and length $\left(\mathcal{O}^{\prime *}\right) \leq$ $\frac{3}{2}\left|G^{\prime}\right|$. The claims are trivially true.

Suppose the claims are true for $j=k$.

Suppose that $\triangle_{k+1}=\left\{a_{k+1}, b_{k+1}, c_{k+1}\right\}$. Without loss of generality, assume the edges of $\triangle_{k+1}$ are oriented in $\mathcal{O}_{k}$ as $\left(a_{k+1} \rightarrow b_{k+1}\right),\left(b_{k+1} \rightarrow c_{k+1}\right),\left(a_{k+1} \rightarrow\right.$ $\left.c_{k+1}\right)$.

By Theorem 1 $G_{\triangle_{k+1}}$ has an st-orientation $\mathcal{O}_{\triangle_{k+1}}$, with $a_{k+1}$ as the source and $c_{k+1}$ as the sink, such that length $\left(\mathcal{O}_{\triangle_{k+1}}\right) \leq\left|G_{\triangle_{k+1}}\right|-1$ and length $\left(\mathcal{O}_{\triangle_{k+1}}^{*}\right) \leq$ $2\left|G_{\triangle_{k+1}}\right|-5$.

We show the orientation $\mathcal{O}_{k+1}=\mathcal{O}_{k} \oplus \mathcal{O}_{\triangle_{k+1}}$ satisfies the claims.

Both the upper bounds of length $\left(\mathcal{O}_{j}\right)$ in Theorem 5 and Lemma 3 can be written in the form

$$
\operatorname{length}\left(\mathcal{O}_{j}\right) \leq \alpha\left|G_{j}\right|+(1-\alpha) \sum_{i=1}^{j}\left|I\left(G_{\triangle_{i}}\right)\right|+\beta .
$$

Since we use an st-orientation with the height at most $\frac{2}{3}\left|G_{0}\right|+14$ in Theorem 5 $\alpha$ is $\frac{2}{3}$ and $\beta$ is 14 . On the other hand, in the base case of this proof, we use an st-orientation with height at most $\frac{3}{4}\left|G_{j}\right|+2\left\lceil\sqrt{\left|G^{\prime}\right|}\right\rceil+4$. By the same process, the value of $\alpha$ is $\frac{3}{4}$ and $\beta$ is $2\left\lceil\sqrt{\left|G^{\prime}\right|}\right\rceil+4$ in this case. Hence the proof of Claim 1 is similar to the proof of Claim 1 in Theorem 5 In the following, we prove Claim 2.

By induction hypothesis, $G_{k}$ has an st-orientation $\mathcal{O}_{k}$ such that length $\left(\mathcal{O}_{k}^{*}\right) \leq$ $\frac{3}{2}\left|G_{k}\right|+\frac{\sum_{i=1}^{k}\left|I\left(G_{\triangle_{i}}\right)\right|}{2}$. Also, we know that length $\left(\mathcal{O}_{\triangle_{k+1}}^{*}\right) \leq 2\left|G_{\triangle_{k+1}}\right|-5$. As in the proof of Theorem 5 , there are two cases for analyzing length $\left(\mathcal{O}_{k+1}^{*}\right)$.

(a) $P_{k+1}^{*}$ does not pass through $f_{k+1}$. Then $P_{k+1}^{*}$ is a path in $G_{k}^{*}$ and the claim trivially holds. 
(b) $P_{k+1}^{*}$ passes through $f_{k+1}$. Then

$$
\begin{aligned}
\operatorname{length}(\mathcal{O}) & \leq \frac{3}{2}\left|G_{k}\right|+\frac{1}{2} \sum_{i=1}^{k}\left|I\left(G_{\triangle_{i}}\right)\right|+2\left|G_{\triangle_{k+1}}\right|-5-1 \\
& =\frac{3}{2}\left|G_{k}\right|+\frac{1}{2} \sum_{i=1}^{k}\left|I\left(G_{\triangle_{i}}\right)\right|+2\left|I\left(G_{\triangle_{k+1}}\right)\right| \\
& =\frac{3}{2}\left|G_{k+1}\right|+\frac{1}{2} \sum_{i=1}^{k+1}\left|I\left(G_{\triangle_{i}}\right)\right|
\end{aligned}
$$

This completes the induction.

Theorem 6 Let $G_{v}$ be a 4-block component of $G$ associated with a node $v$ of the 4-block tree of $G$, and let $\triangle_{v}$ be the separating triangle in $G$ corresponding to node $v$. Then $G$ has an st-orientation $\mathcal{O}$ such that length $(\mathcal{O}) \leq \frac{3}{4} n+\frac{1}{4}(n-$ $\left.\left|G_{v}\right|\right)+2\left\lceil\sqrt{\left|G_{v}\right|}\right\rceil+5$ and length $\left(\mathcal{O}^{*}\right) \leq \frac{3}{2} n+\frac{n-\left|G_{v}\right|}{2}$.

Proof: Let $S=\left\{\triangle_{1}, \triangle_{2}, \ldots, \triangle_{h}\right\}$ be the set of maximal separating triangles of $G_{\triangle_{v}}$. Since $G_{v}$ is 4-connected, by Lemma 3. $G_{\triangle_{v}}$ has an st-orientation $\mathcal{O}_{\triangle_{v}}$ such that

$$
\begin{aligned}
\operatorname{length}\left(\mathcal{O}_{\triangle_{v}}\right) & \leq \frac{3}{4}\left|G_{\triangle_{v}}\right|+2\left\lceil\sqrt{\left|G_{v}\right|}+4+\frac{\sum_{i=1}^{h}\left|I\left(G_{\triangle_{i}}\right)\right|}{4}\right. \\
\operatorname{length}\left(\mathcal{O}_{\triangle_{v}}^{*}\right) & \leq \frac{3}{2}\left|G_{\triangle_{v}}\right|+\frac{\sum_{i=1}^{h}\left|I\left(G_{\triangle_{i}}\right)\right|}{2} .
\end{aligned}
$$

Let $G_{e x t}=G-I\left(G_{\triangle_{v}}\right)$. By Theorem 1, $G_{e x t}$ has an st-orientation such that $\operatorname{length}\left(\mathcal{O}_{\text {ext }}\right) \leq\left|G_{\text {ext }}\right|-1$ and length $\left(\mathcal{O}_{\text {ext }}^{*}\right) \leq 2\left|G_{\text {ext }}\right|-5$. Let $\mathcal{O}=\mathcal{O}_{\text {ext }} \oplus \mathcal{O}_{\triangle_{v}}$. Then

$$
\begin{aligned}
\operatorname{length}(\mathcal{O}) & \leq \operatorname{length}\left(\mathcal{O}_{\text {ext }}\right)+\operatorname{length}\left(\mathcal{O}_{\triangle_{v}}\right)-1 \\
& \leq\left(\left|G_{\text {ext }}\right|-1\right)+\frac{3}{4}\left|G_{\triangle_{v}}\right|+2\left\lceil\sqrt{\left|G_{v}\right|}+4+\frac{\sum_{i=1}^{h}\left|I\left(G_{\triangle_{i}}\right)\right|}{4}-1\right. \\
& =\frac{3}{4}\left|G_{\text {ext }}\right|+\frac{1}{4}\left|G_{e x t}\right|+\frac{3}{4}\left|G_{\triangle_{v}}\right|+2\left\lceil\sqrt{\left|G_{v}\right|}\right\rceil+\frac{\sum_{i=1}^{h}\left|I\left(G_{\triangle_{i}}\right)\right|}{4}+2 \\
& =\frac{3}{4}(|G|+3)+\frac{1}{4}\left(\left|V\left(G_{e x t}\right) \cup\left(\bigcup_{i=1}^{h} I\left(G_{\triangle_{i}}\right)\right)\right|\right)+2\left\lceil\sqrt{\left|G_{v}\right|}\right\rceil+2 \\
& =\frac{3}{4}(n+3)+\frac{1}{4}\left(n-\left|G_{v}\right|+3\right)+2\left\lceil\sqrt{\left|G_{v}\right|}\right\rceil+2 \\
& =\frac{3}{4} n+\frac{1}{4}\left(n-\left|G_{v}\right|\right)+2\left\lceil\sqrt{\left|G_{v}\right|}\right\rceil+5
\end{aligned}
$$


and

$$
\begin{aligned}
\operatorname{length}\left(\mathcal{O}^{*}\right) & =\operatorname{length}\left(\mathcal{O}_{\text {ext }}^{*}\right)+\operatorname{length}\left(\mathcal{O}_{\triangle_{v}}^{*}\right)-1 \\
& \leq\left(2\left|G_{\text {ext }}\right|-5\right)+\left(\frac{3}{2}\left|G_{\triangle_{v}}\right|+\frac{\sum_{i=1}^{h}\left|I\left(G_{\triangle_{i}}\right)\right|}{2}\right)-1 \\
& =\frac{3}{2}\left|G_{\text {ext }}\right|+\frac{3}{2}\left|G_{\triangle_{v}}\right|+\frac{1}{2}\left|G_{\text {ext }}\right|+\frac{\sum_{i=1}^{h}\left|I\left(G_{\triangle_{i}}\right)\right|}{2}-6 \\
& =\frac{3}{2}(|G|+3)+\frac{1}{2}\left(\left|I\left(G_{\text {ext }}\right) \cup\left(\bigcup_{i=1}^{h} I\left(G_{\triangle_{i}}\right)\right)\right|+3\right)-6 \\
& =\frac{3}{2} n+\frac{1}{2}\left(n-\left|G_{v}\right|\right)
\end{aligned}
$$

This completes the proof.

Theorem 7 Every plane triangulation $G$ of $n$ vertices has a VR with height $\leq \frac{23}{24} n+2\lceil\sqrt{n}\rceil+10$ and width $\leq \frac{23}{12} n$. The VR can be constructed in linear time.

Proof: By Lemma 2, there are two cases.

Case 1: $G$ has a 4 -block component with size $n_{1} \geq n / 6$. By Theorem 6 , $G$ has an st-orientation $\mathcal{O}$ such that length $(\mathcal{O}) \leq \frac{3}{4} n+\frac{n-n_{1}}{4}+2\lceil\sqrt{n}\rceil+5$ and length $\left(\mathcal{O}^{*}\right) \leq \frac{3 n}{2}+\frac{\left(n-n_{1}\right)}{2}$. Since $n_{1} \geq \frac{n}{6}$, we have

$$
\begin{aligned}
\operatorname{length}(\mathcal{O}) & \leq \frac{23}{24} n+2\lceil\sqrt{n}\rceil+5 \\
\operatorname{length}\left(\mathcal{O}^{*}\right) & \leq \frac{23}{12} n
\end{aligned}
$$

Case 2: $G$ has a set of unrelated separating triangles $\left\{\triangle_{1}, \triangle_{2}, \ldots, \triangle_{h}\right\}$ such that

- For all $i,\left|G_{\triangle_{i}}\right| \geq 5$, (which implies $\left.\left|I\left(G_{\triangle_{i}}\right)\right| \geq 2\right)$.

- $\frac{n}{4}-3 \leq \sum_{i=1}^{h}\left|I\left(G_{\triangle_{i}}\right)\right| \leq \frac{3}{4} n-3$.

Since $\mathcal{X}(z) \geq z / 3$ for all $z \geq 2$, we have

$$
\sum_{i=1}^{h} \mathcal{X}\left(\left|I\left(G_{\triangle_{i}}\right)\right|\right) \geq \frac{\sum_{i=1}^{h}\left|I\left(G_{\triangle_{i}}\right)\right|}{3}
$$

By Theorem [5, $G$ has an st-orientation $\mathcal{O}$ such that 


$$
\begin{aligned}
\operatorname{length}\left(\mathcal{O}^{*}\right) & \leq 2 n-5-\sum_{i=1}^{h} \mathcal{X}\left(\left|I\left(G_{\triangle_{i}}\right)\right|\right) \\
& \leq 2 n-5-\frac{\left.\sum_{i=1}^{h} \mid I\left(G_{\triangle_{i}}\right)\right)}{3} \leq 2 n-5-\frac{n / 4-3}{3}<\frac{23}{12} n \\
\operatorname{length}(\mathcal{O}) & \leq \frac{2 n}{3}+\frac{\sum_{i=1}^{h}\left|I\left(G_{\triangle_{i}}\right)\right|}{3}+14 \\
& \leq \frac{2 n}{3}+\frac{3 n / 4-3}{3}+14=\frac{11}{12} n+13 \\
& \leq \frac{23}{24} n+2\lceil\sqrt{n}\rceil+10
\end{aligned}
$$

The last inequality holds provided $n \geq 3$. In either case, the orientation $\mathcal{O}$ leads to a VR of $G$ with the stated width and height.

To construct the VR, we first find the decomposition in Lemma 2, which can be done in linear time. Then we use the VR constructions in Theorems 1 , 2. 3 and 4 for different 4-block components of $G$. Since all these VRs can be constructed in linear time, our algorithm also takes linear time.

\section{Conclusion}

In this paper, we showed that every plane graph of $n$ vertices has a VR with height $\leq \frac{23}{24} n+2\lceil\sqrt{n}\rceil+10$ and width $\leq \frac{23}{12} n$. This is the first VR construction for general plane graphs that simultaneously bounds the height and the width away from the trivial upper bound. The gap between the size of our VR and the known lower bound is still large. It would be interesting to find more compact VR constructions. 


\section{References}

[1] C. Chen, Y. Hung, and H. Lu. Visibility representations of four connected plane graphs with near optimal heights. In Proc. 17th International Symposium on Graph Drawing, volume 5417 of LNCS. Springer, 2009.

[2] P. O. de Mendez. Orientations bipolaires. PhD thesis, École des Hautes Études en Sciences Sociales, Paris, 1994.

[3] G. Di Battista, P. Eades, R. Tammassia, and I. Tollis. Graph drawing: Algorithms for the visualization of graphs. Prentice Hall, 1999.

[4] J. Fan, C. Lin, H. Lu, and H. Yen. Width-optimal visibility representations of plane graphs. In 18th International Symposium on Algorithms and Computation, volume 4835 of LNCS, pages 160-171. Springer, 2007.

[5] X. He, J.-J. Wang, and H. Zhang. Compact visibility representation of 4connected plane graphs. In 4th Annual International Conference on Combinatorial Optimization and Applications, volume 6508 of LNCS, pages 339353. Springer, 2010.

[6] X. He and H. Zhang. Nearly optimal visibility representations on plane graphs. In 33rd International Colloquium on Automata, Languages and Programming, volume 4051 of LNCS, pages 407-418. Springer, 2006.

[7] G. Kant. A more compact visibility representation. International Journal of Computational Geometry and Applications, 7:197-210, 1997.

[8] G. Kant and X. He. Regular edge labeling of 4-connected plane graphs and its applications in graph drawing problems. Theoretical Computer Science, 172:175-193, 1997.

[9] A. Lempel, S. Even, and I. Cederbaum. An algorithm for planarity testing of graphs. In Theory of Graphs: International Symposium, pages 215-232, 1967.

[10] C.-C. Lin, H.-I. Lu, and I.-F. Sun. Improved compact visibility representation of planar graph via schnyder's realizer. SIAM Journal on Discrete Mathematics, 18:19-29, 2004.

[11] C. Papamanthou and I. G. Tollis. Applications of parameterized storientations in graph drawing algorithms. In Proc. 13th International Symposium on Graph Drawing, volume 3843 of LNCS, pages 355-367. Springer, 2005.

[12] C. Papamanthou and I. G. Tollis. Parameterized st-orientations of graphs: Algorithms and experiments. In Proc. 14th International Symposium on Graph Drawing, volume 4372 of LNCS, pages 220-233. Springer, 2006.

[13] P. Rosenstiehl and R. E. Tarjan. Rectilinear planar layouts and bipolar orientations of planar graphs. Discrete Comput. Geom., 1:343-353, 1986. 
334 Wang et al. VR of Plane Graphs with Simultaneous Bound

[14] R. Tamassia and I.G.Tollis. A unified approach to visibility representations of planar graphs. Discrete Comput. Geom., 1:321-341, 1986.

[15] H. Zhang and X. He. An application of well-orderly trees in graph drawing. In Proc. 13th International Symposium on Graph Drawing, volume 3843 of LNCS, pages 458-467. Springer, 2005.

[16] H. Zhang and X. He. Canonical ordering trees and their applications in graph drawing. Discrete Comput. Geom., 33:321-344, 2005.

[17] H. Zhang and X. He. Improved visibility representation of plane graphs. Computational Geometry: Theory and Applications, 30:29-39, 2005.

[18] H. Zhang and X. He. Visibility representation of plane graphs via canonical ordering tree. Information Processing Letters, 96:41-48, 2005.

[19] H. Zhang and X. He. Optimal st-orientations for plane triangulations. $J$. Comb. Optim., 17:367-377, 2009. 